\title{
ON SOME MATHEMATICAL ASPECTS OF KINETIC MODEL EQUATIONS*
}

\author{
BY \\ LAWRENCE SIROVICH (Brown University) \\ AND \\ JAMES K. THURBER (Brookhaven National Laboratories)
}

\begin{abstract}
A class of integro-differential equations used in the kinetic theory of gases is investigated. The existence and uniqueness of solutions to the initial value problem associated with these equations is proved under relatively weak conditions. Under somewhat stronger assumptions the validity of the application of FourierLaplace transforms to this problem is demonstrated. Finally the asymptotic approach to hydrodynamics is shown.

1. Introduction. The development of the kinetic theory of gases has been slow due to the formidable nature of the Boltzmann equation. Recently considerable progress in kinetic theory has been made by use of certain "ersatz" Boltzmann equations [1], [2], [3]. These equations retain many of the essential features of the Boltzmann equation but are less formidable mathematically. These "ersatz" equations called model equations, like the Boltzmann equation, are integro-differential equations. In the present paper we shall demonstrate that the initial value problem for the linearized forms of these equations is well-posed, i.e. we shall show the existence and uniqueness of solutions, and further that these depend continuously on the initial data. This is shown for all times under mild restrictions on the initial data.

It is also proven that the solution to the initial value problem can be obtained explicitly by first applying Fourier transforms in the space variables followed by the application of the Laplace transform in the time variable. Using this form of the solution we then demonstrate that the solutions are uniformly bounded in time. A major result of this paper is the stronger conclusion, that the solutions to the model equations behave asymptotically as the solutions of the equations of hydrodynamics.

The present mathematical study is part of a series of investigations of kinetic models [4]-[7] which the authors have made. The above results were first discussed in our report [4]. Also we have made an intensive analytical and numerical analysis of free and forced soundwaves using the kinetic models. Exceptional agreement has been found with experiment [7] for the full range of existing experimental data.

These and other investigations demonstrate the importance of model equations in kinetic theory. The present investigation indicates that kinetic models are satisfactory from the mathematical point of view. It has been shown in a formal theory that the Boltzmann equation asymptotically behaves as the equations of hydrodynamics [8][10]. A comparison of the present results with the latter shows that by choosing a fine

\footnotetext{
*Received June 4, 1966; revised manuscript received August 19, 1966. The results contained in this paper were obtained in the course of research sponsored by National Science Foundation under Grant GP 3753, and under the auspices of the U. S. Atomic Energy Commission. The revised version of this paper was prepared under ONR 562(39).
} 
enough model one may have as much identification with the formal asymptotic solution of the Boltzmann equation as one desires. This of course leads to further confidence in the model equations.

2. The initial value problem. In kinetic theory one is interested in obtaining the mass distribution function [11]

$$
f=f(x, \xi, t)
$$

defined such that $f d \mathbf{x} d \xi$ gives the mass of particles at position $\mathbf{x}$ with velocity $\xi$ at time $t$ in the volume $d \mathbf{x} d \xi$ of phase space. In equilibrium this is given by

$$
f=f^{0}=\frac{\rho_{0}}{\left(2 \pi R T_{0}\right)^{3 / 2}} \exp \left[-\xi^{2} / 2 R T_{0}\right]
$$

where $\rho_{0}, T_{0}$ and $R$ are the mass density, temperature and the gas constant respectively. For small departures from equilibrium one is interested in the perturbed distribution function,

$$
g=\left(f-f^{0}\right) / f^{0}
$$

which satisfies the equation

$$
(\partial / \partial t+\xi \cdot \partial / \partial \mathrm{x}) g=L(g)
$$

where $L$ denotes the linearized collision operator of the Boltzmann equation [11].

It has been found useful in kinetic theory to examine "ersatz" Boltzmann equations of the form

$$
\left(\frac{\partial}{\partial t}+\xi \cdot \frac{\partial}{\partial \mathbf{x}}+1\right) g=\sum_{m, n=0}^{N} \lambda_{m n} a_{m} \phi_{n}
$$

where the symbols occurring on the right hand side will be defined presently. We assume that in (5), $t, \xi$ and $\mathbf{x}$ have been suitably nondimensionalized. The $\phi_{n}$ are polynomials in $\xi$ and are defined as follows

with

$$
\phi_{n}=S_{l+1 / 2}^{(r)}\left(\xi^{2} / 2\right)|\xi|^{l} P_{l}^{m}(\cos \theta) \exp (\operatorname{im} \chi) /\left(N_{r l m}\right)^{1 / 2}
$$

$$
N_{r l m}=\frac{2^{l+1} \Gamma(r+l+3 / 2)(l+|m|) !}{\pi^{1 / 2} r !(2 l+1)(l-|m|) !} .
$$

In (6), $\theta$ and $\chi$ denote angular variables in spherical velocity space, and $S_{l+1 / 2}^{r}$, $P_{l}^{m}$ are the Sonine and Legendre polynomials respectively [12]. It has been assumed in (6) that the array $r, l, m$ has been reduced to a one-dimensional array $(n)$. This of course can be done in a variety of ways and the precise way in which it is done will have no bearing until Sec. 4 .

The $\lambda_{m n}$ in (5) are real dimensionless constants which are derived from the exact linear collision operator, $L$. They depend on the intermolecular force law of the gas. However, in the present context, they need not be specified. (In the last section only certain properties of the $\lambda_{m n}$ will be needed.)

We define the dimensionless Maxwellian

$$
\omega=\frac{1}{(2 \pi)^{3 / 2}} \exp \left[-\xi^{2} / 2\right],
$$


and the inner product

$$
(g, f)=\int g f^{c} \omega d \xi,
$$

where the superscript $c$ denotes the complex conjugate. One $m^{n} y$ show that

$$
\left(\phi_{n}, \phi_{m}\right)=\delta_{n m} \text {. }
$$

$a_{m}$ of (5) are given by

$$
a_{m}=a_{m}(\mathrm{x}, t)=\left(g, \phi_{m}\right) .
$$

In place of (5) we find it more convenient to treat them entent equation

$$
\frac{\partial G}{\partial t}+\xi \cdot \frac{\partial G}{\partial \mathrm{x}}=\sum_{m, n=0}^{N} \lambda_{m n} A_{n} \phi_{i}
$$

with

and

$$
G=e^{t} g
$$

$$
A_{m}=e^{i} a_{m}=\left(G, \phi_{m}\right) .
$$

We denote the initial data of the problem by

$$
G(\mathrm{x}, \xi, t=0)=G_{0}(\mathrm{x}, \xi)=g(\mathrm{x}, \xi, t=0) .
$$

We shall show that the initial value problem as depicted by Eqs. (11) and (13) with initial data (14) constitutes a well-posed problem.

We first prove the existence, uniqueness and membership in $C^{1}$ in $\mathbf{x}$ and $t$ of solutions to the related integral equation obtained from (11) by formal integration

$$
G=G_{0}(\mathrm{x}-\xi t, \xi)+\int_{0}^{t} \sum_{m, n=0}^{N} A_{m}\left(\mathrm{x}^{*}, s\right) \lambda_{m n} \phi_{n} d s
$$

where

$$
A_{m}(\mathbf{x}, t)=\left(G, \phi_{m}\right)
$$

and

$$
\mathbf{x}^{*}=\mathbf{x}-\xi(t-s) .
$$

Let $S$ denote the space of all functions $F=F(x, t)$ such that $\partial F / \partial x_{i}(j=1,2,3)$ exist and are continuous functions of $t$ and $\mathbf{x}$ for all $\mathbf{x}$ and for all $t \geq 0$. We further specify $\mathcal{S}$ by requiring that for each $F$ there exists a continuous function $M(t)$, such that $|F|$, $\left|\partial F / \partial x_{i}\right| \leq M(t)$ for all $t \geq 0$. Next we introduce the space $S^{N+1}$ which is the $N$-fold direct product of $\delta$ with itself. Let $G_{0}(\mathbf{x}, \xi) \varepsilon C^{1}$ in $\mathbf{x}$ and $C^{0}$ in $\xi$, be such that $G_{0}$ and $\partial G_{0} / \partial x_{i}=O\left(\exp \left[|\xi|^{\alpha}\right]\right)$ uniformly in $\mathrm{x}$ for some $\alpha<2$. The following relations then define a mapping $\mathcal{L}$ of $\delta^{N+1}$ into $\delta^{N+1}$.

$$
\begin{gathered}
\mathcal{S}=G_{0}(\mathrm{x}-\xi t, \xi)+\int_{0}^{t} \sum_{m, n=0}^{N} F_{m}\left(\mathrm{x}^{*}, s\right) \lambda_{m n} \phi_{n} d s, \\
\mathscr{g}_{m}=\left(\mathcal{G}, \phi_{m}\right) \text { for } 0 \leq m \leq N, \\
\partial \mathcal{S}_{m} / \partial x_{i}=\left(\partial \mathcal{S} / \partial x_{i}, \phi_{m}\right) \text { for } 0 \leq m \leq N \text { and } j=1,2,3 .
\end{gathered}
$$


Although $\left(18^{\prime \prime}\right)$ is a consequence of $\left(18^{\prime}\right)$ we regard it as part of our mapping.

It is convenient to write

$$
\mathbf{F}=\left(F_{0}, \partial F_{0} / \partial x_{1}, \partial F_{0} / \partial x_{2}, \partial F_{0} / \partial x_{3}, \cdots, F_{n}, \partial F_{N} / \partial x_{1}, \partial F_{N} / \partial x_{2}, \partial F_{N} / \partial x_{3}\right)
$$

and define

Also we refer to

$$
|\mathrm{F}|_{x}=\sum_{n=0}^{N}\left\{\max _{x}\left|F_{n}\right|+\sum_{i=1}^{3} \max _{x}\left|\frac{\partial F_{n}}{\partial x_{j}}\right|\right\}
$$

$$
\left(F_{m-1}, \partial F_{m-1} / \partial x_{1}, \partial F_{m-1} / \partial x_{2}, \partial F_{m-1} / \partial x_{3}\right)
$$

as the $m$ th component of $\mathrm{F}$. We denote the mapping $(18),\left(18^{\prime}\right),\left(18^{\prime \prime}\right)$ by $\mathcal{L}$ and write

$$
\mathfrak{I}=\mathfrak{L}(\mathbf{F}) \text {. }
$$

We shall also employ

$$
\|F\|_{\iota_{\bullet}}=\max _{0 \leq t \leq t_{\bullet}}|F|_{x}
$$

as a norm in $s^{N+1}$.

We now show that there exists a positive constant $T$ such that for $(t \mid 0 \leq t \leq T)$ the above mapping $\mathcal{L}$ is a contracting mapping. That is

$$
\left\|\mathscr{L}(\mathrm{F})-\mathscr{L}\left(\mathrm{F}^{\prime}\right)\right\|_{T} \leq C\left\|\mathrm{~F}-\mathrm{F}^{\prime}\right\|_{\boldsymbol{T}}
$$

where $C$ is a positive constant less than one and which depends only on the functions $\phi_{n}$ and the constants $\lambda_{m n}$ and $T$.

To see this we note that there exists a constant $P$ such that

$$
\lambda \sum_{m, n}^{N}\left(\left|\phi_{n}\right|,\left|\phi_{m}\right|\right) \leq P
$$

where

$$
\lambda=\max _{n, m \leq N}\left|\lambda_{m n}\right| .
$$

This implies that

$$
\left\|\mathfrak{L}(\mathbf{F})-\mathfrak{L}\left(\mathbf{F}^{\prime}\right)\right\| \leq \int_{0}^{\imath} P\left\|\mathbf{F}-\mathbf{F}^{\prime}\right\|_{\imath} d s=t P\left\|\mathbf{F}-\mathbf{F}^{\prime}\right\|_{\imath} .
$$

It is immediate that there exists a positive $T$ such that $0<C=P T<1$ and for $t \leq T$ we have a contracting mapping. Hence by the well known contracting mapping theorem we know that there exists a unique fixed point of the mapping for the interval $0 \leq t \leq T$. We will denote this point by $F^{0}$.

Next we use $F^{0}$ to define

$$
G_{0}^{*}=G_{0}(\mathbf{x}-\xi t, \xi)+\int_{0}^{t} \sum_{m, n=0}^{N} F_{m}^{0}\left(\mathbf{x}^{*}, s\right) \lambda_{m n} \phi_{n} d s
$$

where $0 \leq t \leq T$. An immediate calculation shows that $G_{0}^{*}$ satisfies (11) through (14). The $F_{n}^{0}$ are to be identified with the $A_{n}$ and the corresponding moments of $G_{0}^{*}$ give the appropriate $F_{n}^{0}$. Further the correct initial condition is clearly satisfied. Thus we have the existence and uniqueness of $G$ for the interval $0 \leq t \leq T$. 
Taking $G_{0}^{*}(\mathbf{x}, \xi, T)$ as our initial value at $t=T$ we repeat the above process and obtain existence and uniqueness of $G$ for the interval $T \leq t \leq 2 T$. This can be done since the same constant $C$ will play the same role and depends only on the fixed constant $P$ and the time interval $T$. Hence we have an existence and uniqueness theorem for $0 \leq t \leq 2 T$. Clearly this can be extended to all $(t \mid 0 \leq t \leq \infty)$.

We also have continuous dependence on the initial data. For if we multiply the initial value $G_{0}$ by $\epsilon$ we obtain $\epsilon G$ for the solution in place of $G$. Since the moments of $G$ are bounded by some continuous function $M(t)$ uniformly in $\mathbf{x}$ we have from (15)

$$
|\epsilon G| \leq \epsilon O\left(\exp \left[|\xi|^{\alpha}\right]\right)+(N+1) \epsilon \int_{0}^{t} M(s) d s O\left(\exp \left[|\xi|^{\alpha}\right]\right)
$$

uniformly in $\mathbf{x}$. Thus for a fixed value of $t$ the solution $\epsilon G$ will approach zero uniformly in $\mathbf{x}$ as $\epsilon \rightarrow 0$. (We may conclude from this that if the initial data is made uniformly small in $\mathbf{x}$ then for a fixed $t, \omega G$ will go to zero uniformly in $\mathbf{x}$.)

3. Fourier and Laplace transforms of the model equations. Now we investigate the legitimacy of taking Fourier and Laplace transforms. We will show that the system (11) through (14) can be solved by first applying the Fourier transform in $\mathbf{x}$, followed by the Laplace transform in $t$. The resulting system is then solved for the transform of $G$ and this operation is followed by Laplace inversion and then by Fourier inversion.

In order to demonstrate the validity of the use of transforms we will assume that the initial distribution $G_{0}$ and its $\mathbf{x}$ gradient are absolutely integrable functions in $\mathbf{x}$. Further we also assume that the Fourier transforms of $G_{0}$ and the components of its gradient are also absolutely integrable in the transform space. As before we take $G_{0}, \nabla G_{0}=$ $O\left(\exp \left[|\xi|^{\alpha}\right]\right)$. From the absolute integrability of $G_{0}$ we have that the Fourier transform of $G_{0}$ is also $O\left(\exp \left[|\xi|^{\alpha}\right]\right)$. As a consequence the moments of $G_{0}, \nabla G_{0}$ and their transforms must also be absolutely integrable. It also follows that the operations of transforms and taking moments of $G_{0}$ are commutative.

Let $|\mathcal{S}|^{N+1}$ denote the subspace of elements of $\delta^{N+1}$ which have the property that all of their components are absolutely integrable and that the Fourier transforms of all of their components are absolutely integrable. The subspace $|\boldsymbol{s}|^{N+1}$ is nonempty since it includes functions in $C^{\infty}$ in $\mathbf{x}$ which are of compact support in $\mathbf{x}$. Then the mapping $\mathfrak{I}=\mathfrak{L}(\mathbf{F})$ given by $(18),\left(18^{\prime}\right),\left(18^{\prime \prime}\right)$ defines a mapping $|\mathcal{S}|^{N+1}$ into $|\mathcal{S}|^{N+1}$. But we have already demonstrated that this mapping has a unique fixed point which must satisfy (11) through (14). Since it also belongs to $|\delta|^{N+1}$ we now know that the usual rules for manipulating with Fourier transforms are correct.

We will use $\mathbf{k}$ to denote the variable corresponding to $\mathbf{x}$ under the Fourier transformation. Denoting the transformed variable by a superscript $T$, the transform of the system (11) through (14) can be written as follows:

$$
\begin{gathered}
\frac{\partial G^{T}}{\partial t}-i \mathbf{k} \cdot \xi G^{T}=\sum_{m, n=0}^{N} \lambda_{m n} A_{m}^{T} \phi_{n}, \\
G^{T}=e^{t} g^{T}, \\
A_{m}^{T}=A_{m}^{T}(\mathbf{k}, t)=\int G^{T} \omega \phi_{m}^{c} d \xi=e^{t} a_{m}^{T}, \\
G_{0}^{T}(\mathbf{k}, \xi)=G(\mathbf{k}, \xi, 0)=g(\mathbf{k}, \xi, 0) .
\end{gathered}
$$

It is more convenient to deal with the following equivalent system: 


$$
\begin{gathered}
\frac{\partial G^{\prime}}{\partial t}=\sum_{m, n=0}^{N} \exp (-i \mathrm{k} \cdot \xi t) \lambda_{m n} A_{m}^{\prime} \phi_{n}, \\
G^{\prime}=\exp (-i \mathrm{k} \cdot \xi t) G^{T}, \\
A_{m}^{\prime}(\mathbf{k}, t)=\left(G^{\prime} \exp (i \mathbf{k} \cdot \xi t), \phi_{m}\right), \\
G_{0}^{\prime}(\mathbf{k}, \xi)=G_{0}^{T}(\mathbf{k}, \xi) .
\end{gathered}
$$

From (30) and (33) we immediately obtain

$$
G^{\prime}=G_{0}^{\prime}(\mathbf{k}, \xi)+\int_{0}^{l} \sum_{m, n=0}^{N} \exp (-i \mathbf{k} \cdot \xi s) \lambda_{m n} A_{m}^{\prime}(\mathbf{k}, s) \phi_{n} d s
$$

Now define

$$
\left\|\mathbf{A}_{i}^{\prime}\right\|=\sum_{n=0}^{N} \max _{\mathbf{k}}\left|A_{n}^{\prime}\right|
$$

and

$$
\left.|| \mathbf{A}^{\prime}\right|_{\imath \bullet} \mid=\max _{0 \leq t \leq \bullet}\left(\sum_{n=0}^{N} \max _{\mathbf{k}}\left|A_{n}^{\prime}\right|\right) .
$$

From (20) and (32) we obtain

$$
\left\|\mathbf{A}_{T}^{\prime}\right\| \leq\left\|\mathbf{A}_{0}^{\prime}\right\|+P \int_{0}^{T}\left\|\mathbf{A}^{\prime}\right\|_{t} d t \leq\left.\right|_{1} \mathbf{A}_{0}^{\prime}\left|+P T \| \mathbf{A}^{\prime}\right|_{T} \mid .
$$

From the continuity of $\left.|| \mathbf{A}^{\prime}\right|_{\ell} \mid$ it follows that

$$
\left((\tau \mid 0 \leq \tau \leq T),\left\|\mathbf{A}_{r}^{\prime}\right\|=\|\left.\mathbf{A}^{\prime}\right|_{\boldsymbol{r}} \mid\right)
$$

Thus

$$
\left.|| \mathbf{A}^{\prime}\right|_{\boldsymbol{T}}\left|=\left\|\mathbf{A}_{\boldsymbol{r}}^{\prime}\right\| \leq\left\|\mathbf{A}_{0}^{\prime}\right\|+\operatorname{Pr}\left\|\left.\mathbf{A}^{\prime}\right|_{\boldsymbol{r}} \leq \leq\right\| \mathbf{A}_{0}^{\prime}\|+P T\| \mathbf{A}^{\prime}\right|_{\boldsymbol{T}} \mid
$$

and consequently

$$
\left.(1-P T)|| \mathbf{A}^{\prime}\right|_{r} \mid \leq\left\|\mathbf{A}_{0}^{\prime}\right\| .
$$

We now choose $T>0$ such that $P T<1$.

Hence

$$
\left.|| \mathbf{A}^{\prime}\right|_{T} \mid \leq\left\|\mathbf{A}_{0}^{\prime}\right\| /(1-P T)
$$

Iterating we obtain

$$
\left.|| \mathbf{A}^{\prime}\right|_{n T}\left|\leq \| \mathbf{A}_{0}^{\prime}\right| \mid /(1-P T)^{n} .
$$

For every $t \geq 0$ there exists an integer $n>0$ such that $t \leq n T$. But

$$
|| \mathbf{A}_{t}^{\prime} \| \leq\left.|| \mathbf{A}^{\prime}\right|_{t}|\leq|\left|\mathbf{A}^{\prime}\right|_{n r} \mid
$$

and hence

$$
\left\|\mathbf{A}_{t}^{\prime}\right\| \leq\left\|\mathbf{A}_{0}^{\prime}\right\| /(1-P T)^{n}
$$

since $0<1-P T<1$, we see that each of the components of $A^{\prime}$ can grow at most exponentially in time. This together with the continuity of $\mathbf{A}^{\prime}$ as a function of $t$ implies 
the validity of the application of Laplace transforms, since from (32) $G^{\prime}$ can grow at most exponentially in time and is clearly continuous. After applying the Laplace transform, the transform of $G$ can be solved for explicitly in terms of the Fourier transform of the initial distribution.

To accomplish this we return to Eqs. (5) and (10). Performing a Fourier transform in space (as above with $\mathrm{k}$ the Fourier variable) and a Laplace transform in time ( $\sigma$, the Laplace variable) and denoting the transformed quantity by a superscript $T$, we obtain,

$$
g^{r}=\frac{g_{0}^{T}}{1+\sigma-i \mathbf{k} \cdot \xi}+\sum_{m, n}^{N} \frac{\lambda_{m n} a_{m}^{r} \phi_{n}}{1+\sigma-i \mathbf{k} \cdot \xi}
$$

where

$$
a_{m}^{T}=\left(\phi_{m}, g^{T}\right) .
$$

$g_{0}^{T}$ is of course the transformed initial data. Division by $(1+\sigma-i \mathbf{k} \cdot \xi)$ in (41) is permissible since $\operatorname{Re} \sigma>0$.

On taking moments (42) we obtain an $(N+1) \times(N+1)$ linear system in the $\boldsymbol{a}_{n}^{\boldsymbol{T}}$. In vector notation this is,

where

$$
(\mathbf{I}-\mathbf{K}) \mathbf{a}^{T}=\mathbf{L}
$$

and

$$
L_{p}=\left(\phi_{p}, g_{0}^{T} /(1+\sigma-i \mathbf{k} \cdot \xi)\right)
$$

$$
K_{p m}=\sum_{n=0}^{N} \lambda_{m n}\left(\phi_{p}, \frac{\phi_{n}}{1+\sigma-i \mathbf{k} \cdot \xi}\right) .
$$

The terms $\left(\phi_{p}, \phi_{n} /(1+\sigma-i \mathbf{k} \cdot \xi)\right)$ can be evaluated explicitly in terms of the complex error function [6].

As we shall show shortly the matrix $(I-K)$ may be inverted for $\operatorname{Re} \sigma>0$. We then have

$$
\mathrm{a}^{T}=(\mathrm{I}-\mathrm{K})^{-1} \mathrm{~L},
$$

and the integral representation for a follows from first inverting the Laplace transform and then the Fourier transform. Finally the solution for $g$ follows by substitution in (41) and a similar inversion.

At this point it is not clear that we can interchange the order of taking and inverting the Fourier and Laplace transforms. This is due to the existence of functions which are such that no multiplier of the form $\exp [-\sigma t](\operatorname{Re} \sigma>0)$ will make the resulting produce absolutely integrable in both $x$ and $t$, whereas for each $t$ they may be absolutely integrable in $x$ and vice versa. For example let

$$
\mathfrak{F}=\exp \left[-|x| \exp \left(-t^{2}\right)\right]
$$

and

$$
\mathfrak{F}^{\prime}=\exp \left[-\sigma t-|x| \exp \left(-t^{2}\right)\right] \quad(\operatorname{Re} \sigma>0) .
$$

Then, for $t \geq 0$ and $-\infty<x<\infty,(46)$ is such a function as can be seen by integrating (47) in $t$ holding $x$ fixed and vice versa. 
We can however say that $g$ or equivalently $G$ is exponentially bounded in time. This can be done by defining the norm

$$
\left\{\left\{\mathrm{F}_{t}\right\}\right\}=\sum_{n=0}^{N} \max _{x}\left|\mathrm{~F}_{n}\right|+\sum_{i=1}^{3} \sum_{n=0}^{N} \max _{x}\left|\frac{\partial \mathrm{F}_{n}}{\partial x_{i}}\right| .
$$

From (18), $\left(18^{\prime}\right),\left(18^{\prime \prime}\right)$ and (22) we have

$$
\left\{\left\{\mathbf{F}_{T}\right\}\right\} \leq\left\{\left\{\mathbf{F}_{0}\right\}\right\}+P \int_{0}^{T}\|\mathbf{F}\|_{t} d t \leq P T\|\mathbf{F}\|_{T} .
$$

We then mimic the analysis (35) through (40) and obtain the exponential boundedness of $G$ and of its moments uniformly in x. However after taking the Laplace transform we do not know that the Laplace transform of $G$ is absolutely integrable in $\mathrm{x}$-space.

The work of this section has proved the existence of a solution to any model equation by means of transforms. An alternate method of accomplishing the same result is by formal techniques. Transforms are then formally taken and subsequently inverted. Finally one proves the convergence of the formal expressions and then one shows that the functions thus obtained satisfy the governing equations. Although the latter portion of such a procedure is quite simple, the former portion proves to be extremely tedious.

4. Boundedness of solutions and their asymptotic approach to hydrodynamics. To obtain additional information on the solutions of model equations a finer analysis is necessary. We first need more specific details about the quantities $\lambda_{r m}$. To specify these, we first define the real quantities (see Eq. (4)).

$$
\beta_{r l}^{(N)}=\frac{\left(L\left(\phi_{r}\right), \phi_{l}\right)}{\left|\left(L\left(\phi_{N+1}\right), \phi_{N+1}\right)\right|}=\beta_{l r}^{(N)} .
$$

The denominator in the above expression is introduced as a normalization and hence the $\beta_{r l}^{(N)}$ vary with the model as is indicated by the superscript. We also take the $\phi_{r}$ ordered according to increasing magnitudes of $\left(L\left(\phi_{r}\right), \phi_{r}\right)$, [8]. One can show that [11]

$$
(L(g), g) \leq 0
$$

so that all diagonal entries $\beta_{\mu \mu}$ of (50) are nonpositive. Further one can also show that [11]

$$
\beta_{r m}=0, \quad r=0,1,2,3,4 \text {. }
$$

(The first five $\phi_{r}$ 's are $\phi_{0} \sim 1, \phi_{1} \sim \xi_{1}, \phi_{2,3} \sim \xi_{2} \pm i \xi_{3}, \phi_{4} \sim \xi^{2}-3$. Equality in (51) holds only if $g=\sum_{r-1}^{4} a_{r} \phi_{r}$.) The $\lambda_{r l}$ are now defined as [3]

$$
\lambda_{r l}=\delta_{r l}+\beta_{r l} \text {. }
$$

Returning to (45) we see that the solution for the $a_{r}$ is given by

$$
\mathbf{a}(\mathbf{x}, t)=\frac{1}{(2 \pi)^{3}} \int_{-\infty}^{\infty} \exp (-i \mathbf{k} \cdot \mathbf{x}) d \mathbf{k} \int \frac{e^{\sigma t} d \sigma}{2 \pi i}(\mathbf{I}-\mathbf{K})^{-1} \mathbf{L} .
$$

The path of integration in the $\sigma$-plane is as usual to the right of the characteristic roots of $(\mathbf{I}-\mathbf{K})$.

In order to discuss these roots we introduce auxiliary quantities $b_{n}$ such that

$$
(\mathbf{I}-\mathrm{K}) \mathrm{b}=0 \text {. }
$$

Regarding $\mathbf{k}$ as given (and real) we can solve for the characteristic roots, $\sigma(\mathbf{k})$, and from 
these obtain the $b_{n}(\mathbf{k})$. This leads us to the auxiliary "distribution function" $q$; defined by

$$
(1+\sigma(\mathbf{k})-i \mathbf{k} \cdot \xi) q=\sum_{n, m=0}^{N} b_{n} \lambda_{n m} \phi_{m} .
$$

Taking the inner product of (56) with $q$ we obtain

$$
\sigma(q, q)+i(\mathbf{k} \cdot \xi q, q)=-(q, q)+\left(\sum_{n, m=0}^{N} b_{n} \lambda_{n m} \phi_{m}, q\right) .
$$

On taking the real part of this equation we obtain

$$
\begin{aligned}
\operatorname{Re} \sigma & =-1+\frac{\left(\sum_{n, m=0}^{N} b_{n} \lambda_{n m} \phi_{m}, q\right)}{(q, q)} \\
& =\frac{\left(\sum_{n, m=0}^{N} b_{n} \beta_{n m}^{(N)} \phi_{m}, \sum_{n=0}^{N} b_{n} \phi_{n}\right)}{(q, q)} \\
& =\frac{\left(L\left(\sum_{n=0}^{N} b_{n} \phi_{n}\right), \sum_{n=0}^{N} b_{n} \phi_{n}\right)}{(q, q)},
\end{aligned}
$$

and from (51) we have that

$$
\sigma(\mathbf{k}) \leq 0 .
$$

As is implied by (52) a necessary and sufficient condition for $\operatorname{Re} \sigma$ to vanish is that $q=\sum_{n=0}^{4} b_{n} \phi_{n}$. Therefore from (51) if $\sigma=0$ then $k=0$. Hence from continuity $\sigma$ is bounded away from zero for $\mathbf{k} \neq 0$. Note that $\mathbf{k}=0$ does not imply that $\sigma$ vanish. In general for $\mathrm{k}=0, \sigma=0$ is a five-fold root as is easily seen from (56), in addition to which there are nonzero roots.

In certain instances $\beta_{r l}$ is diagonal. Molecules which interact with the inverse fifth power of relative distance is an example), and in this case $-1 \leq \sigma \leq 0$. In general it is clear from (56) that $\sigma$ is always bounded from below. From this one may also show that $\mathbf{k}$ is also bounded. However since we may specify initial data arbitrarily this seems to be absurd, and requires some explanation.

An alternate way of obtaining (56) is to ask the question, "Is it possible to have plane wave solutions?" i.e., solutions of the form

$$
q(\xi) \exp [\sigma t-i \mathbf{k} \cdot \mathbf{x}]
$$

The above argument states that a necessary condition is that the magnitude of the wave-number, $|\mathbf{k}|$, must fall in some bound. Moreover the attenuation factor $\operatorname{Re} \sigma$ is bounded above by zero and below by a quantity representative of $\beta_{r l}$. For $\mathbf{k}$ outside the aforementioned bounded regions, the auxiliary problem, (55), is no longer appropriate. For it is seen from (47) that two different analytic functions are obtained depending on the sign of $\operatorname{Im}[(1+\sigma) / i k]$. (This has been discussed elsewhere [4], [6].) However in moving the Bromwich path in the $\sigma$-plane only the analytic continuation of this function can be considered. This then produces roots $\sigma(k)$ for which the denominator in (54) vanishes [5]. But from the above discussion these do not lead to plane waves. (Any attempt at forcing a plane wave solution in this case results in an infinite sum of plane waves which cannot be further reduced.) 
All further discussion will be restricted to one-dimensional motions. In this case $\partial / \partial y=\partial / \partial z=0$ and the reduced set of functions

$$
\psi_{r l}=S_{l+1 / 2}^{r}\left(\frac{\xi^{2}}{2}\right) \xi^{l} P_{l}\left(\frac{\xi_{1}}{\xi}\right)
$$

are now sufficient for a description.

From the properties of Legendre polynomials $P_{l}$, it follows that the $\psi_{r}$ are either odd or even in $\xi_{1}$. Therefore under an elementary change of variable

$$
\left(\psi_{r}, \frac{\psi_{j}}{1+\sigma-i k \xi_{1}}\right)=(\operatorname{sgn} k)^{p}\left(\psi_{r}, \frac{\psi_{i}}{1+\sigma-i|k| \xi_{1}}\right) .
$$

The exponent of $\operatorname{sgn} k, p$, is either 0 or 1 depending on whether the product $\psi_{r} \psi_{j}$ is either even or odd in $\xi_{1}$. From parity considerations (see Appendix IV of [6]) one easily sees that if $\operatorname{sgn} k$ occurs in $\operatorname{det}(\mathbf{I}-\mathbf{K})$ it occurs only as a factor.

Next we observe that if $\operatorname{Re}(\sigma+1)$ changes sign in $\left(\psi_{r}, \psi_{i} /\left(1+\sigma-i|k| \xi_{1}\right)\right)$ a different analytic function is defined. But since $\operatorname{Re} \sigma>0$ in (54) such a change in sign occurs only by virtue of a path change in the $\sigma$-plane. Therefore only the analytic continuation of $\left(\psi_{r}, \psi_{i} /\left(1+\sigma-i|k| \xi_{1}\right)\right)$ can be taken when $\operatorname{Re}(\sigma+1)$ changes sgn. Also since $|k|$ is always positive we are at liberty to define $\left(\psi_{r}, \psi_{i} /\left(1+\sigma-i|k| \xi_{1}\right)\right)$ to be the analytic continuation for nonpositive values of the variables $|k|$. Therefore $\operatorname{det}(1-K)$ modulo a possible factor of $\operatorname{sgn} k$ is an analytic function of the variables $\sigma$ and $|k|$. Hence by the implicit function theorem there are three (for one-dimensional motions there are only three zeros of $\sigma$ for $k=0$ ) distinct roots $\sigma(|k|)$ in the neighborhood of $(\sigma=0,|k|=0)$. Alternately there exists a positive $k_{0} \neq 0$ such that for $|k|<k_{0}$ there are three and only three roots $\sigma_{1}(|k|), \sigma_{2}(|k|), \sigma_{3}(|k|)$ in the neighborhood of the origin. A previous argument then shows that they are the only roots for which $\operatorname{Re} \sigma$ is the neighborhood of the origin.

Considering the one-dimensional form of (54) we write

$$
a(x, t)=a^{(1)}+a^{(2)}
$$

with

$$
\mathrm{a}^{(1)}=\frac{1}{2 \pi} \int_{|k|<k_{0}} \exp \left(-i k_{1} x\right) d k_{1} \int \frac{e^{\sigma t} d \sigma}{2 \pi i}(\mathbf{I}-\mathbf{K}) \mathbf{L}
$$

and

$$
\mathbf{a}^{(2)}=\frac{1}{2 \pi} \int_{|k| \geq k_{0}} \exp \left(-i k_{1} x\right) d k_{1} \int \frac{e^{o t} d \sigma}{2 \pi i}(\mathbf{I}-\mathbf{K}) \mathbf{L} .
$$

Since $|k| \geq k_{0}$ in $\mathrm{a}^{(2)}$ the Bromwich path may be distorted so that $\operatorname{Re} \sigma<0$ along it and is easily shown $a^{(2)}$ is exponentially decreasing in time. Regarding $a^{(1)}$ we may carry out the integration in the $\sigma$-plane so that

$$
a^{(1)}=\frac{1}{2 \pi} \int_{\left|k_{1}\right|<k_{0}} \exp \left(-i k_{1} x\right) \sum_{i=1}^{3} \exp \left(\sigma_{r}\left(k_{1}\right) t\right) 1_{i}\left(k_{1}\right) d k_{1} .
$$

The $l_{i}$ represent the residues of the three poles. From the previously derived properties of the $\sigma_{r}$, simple estimates show that a is bounded in time. A similar discussion for the three-dimensional case can also be given. 
The expression (65) may be used to construct the time asymptotic form of the solution. To obtain this we regard a typical term of (65)

$$
I=\int_{-\infty}^{\infty} \exp \left(-i k_{1} x+\sigma\left(k_{1}\right) t\right) h\left(k_{1}\right) d k_{1}
$$

where $h\left(k_{1}\right)$ is of compact support. The exponent, $\sigma$, satisfies the following conditions

$$
\begin{aligned}
& R \sigma \leq 0, \\
& R \sigma<0, \quad k_{1} \neq 0, \\
& \sigma=i \alpha k+\beta k^{2}+O\left(k^{3}\right) \\
& \beta<0 .
\end{aligned}
$$

These follow immediately from earlier considerations. Under (67) it has shown that the integral (66) has the following asymptotic form [13]

$$
I \sim \int_{-\infty}^{\infty} \exp \left(-i k_{1} x+i \alpha k_{1} t+\beta k_{1}^{2} t\right) h(0) d k_{1}
$$

which is easily integrated.

Rather than give the explicit solution we refer the reader to [13] where the asymptotic form of the initial value problem for the Navier-Stokes equations is carried out. The Taylor series expansions for the model equations have been given elsewhere [4], [5], and one has only to compare this with the comparable Navier-Stokes theory to see that to lowest order the long time behavior is hydrodynamical. Higher orders may be found by carrying further terms in the Taylor expansions [13]. The result of this is fairly interesting for two reasons. First one soon recognizes that the expansions are identical to those found from the Chapman-Enskog equations. Secondly, that the ChapmanEnskog theory does not furnish the correct asymptotic solutions, in general. One finds that asymptotic initial data must be employed with the Chapman-Enskog procedure in order to obtain the correct asymptotic solution. Since these points are discussed for the Boltzmann equation elsewhere [9], [10], we will not go into further detail. We mention however that in contrast to the work of the last reference, the present work is on a mathematically rigorous foundation.

\section{REFERENCES}

1. P. L. Bhatnager, E. P. Gross and M. Krook, Phys. Rev. 94, 511 (1954)

2. E. P. Gross and E. A. Jackson, Phys. Fluids 2, 432 (1959)

3. L. Sirovich, Phys. Fluids 5, 908 (1962)

4. L. Sirovich and J. K. Thurber, Sound propagation according to kinetic models, AFOSR-1380, MF-17, IMS, NYU (1961)

5. L. Sirovich and J. K. Thurber, in Rarefied gas dynamics, Academic Press, New York, (1963)

6. L. Sirovich and J. K. Thurber, in Rarefied gas dynamics, Academic Press, New York and London, (1965)

7. L. Sirovich and J. K. Thurber, J. Acoust. Soc. Am. 37, 329 (1965)

8. L. Sirovich, Phys. Fluids 6, 10 (1963)

9. L. Sirovich, Phys. Fluids 6, 218 (1963)

10. L. Sirovich, Phys. Fluids 6, 1428 (1963) 
11. S. Chapman and T. G. Cowling, The maintmatical theory of non-uniform gases, Cambridge Univ. Press, London and New York, (1952)

12. W. Magnus and F. Oberlettinger, Formulas and theorems for the functions of mathematical physics, Chelsea Publ. Co., New York, 1954

13. L. Sirovich, Initial and boundary value problems in dissipative gas dynamics, (submitted for publication) 